\title{
PANCREAS
}

\section{Cortical thickness-a useful indicator in painful chronic pancreatitis?}

New findings suggest that cortical thickness is an indicator of pain system dysfunction in patients with chronic pancreatitis who experience pain. "We found reduced cortical thickness in brain areas involved in the processing of visceral pain," explains first author Jens Brøndum Frøkjær. "The cortical thinning found in these areas was correlated to the patients' clinical pain score," he continues.

Patients with chronic pancreatitis most commonly present with repeated episodes of abdominal pain. This pain has already been linked with altered microstructures in the brain areas involved in pain processing, but these neuroplastic changes have not been examined in detail.

In this study, Frøkjær et al. used 3D MRI scanning to analyze the brain morphology of 19 patients with chronic pancreatitis who experienced pain and 15 healthy individuals as controls.

Overall, patients with painful chronic pancreatitis had reduced cortical thickness compared with healthy controls $(1.98 \mathrm{~mm}$ versus $2.11 \mathrm{~mm}$, respectively; $P=0.0012$ ). Moreover, this reduction in thickness was observed in areas already known to be involved in visceral pain processing (such as the secondary somatosensory cortex and the frontal cortex) and correlated with patients' average daily pain scores.

"Cortical thickness may, as the end result of long-standing ongoing pain input to the neuromatrix, serve as a valid measure of the overall damage and dysfunction of the pain system," concludes Frøkjær. He hopes that further work using new technologies will help establish other biomarkers of disordered pain processing, which could be used to evaluate new pain-relieving treatments.

\section{Katrina Ray}

Original article Frøkjær, J. B. et al. Reduced cortical thickness of brain areas involved in pain processing in patients with chronic pancreatitis. Clin. Gastroenterol. Hepatol. doi:10.1016/j.cgh.2011.11.024

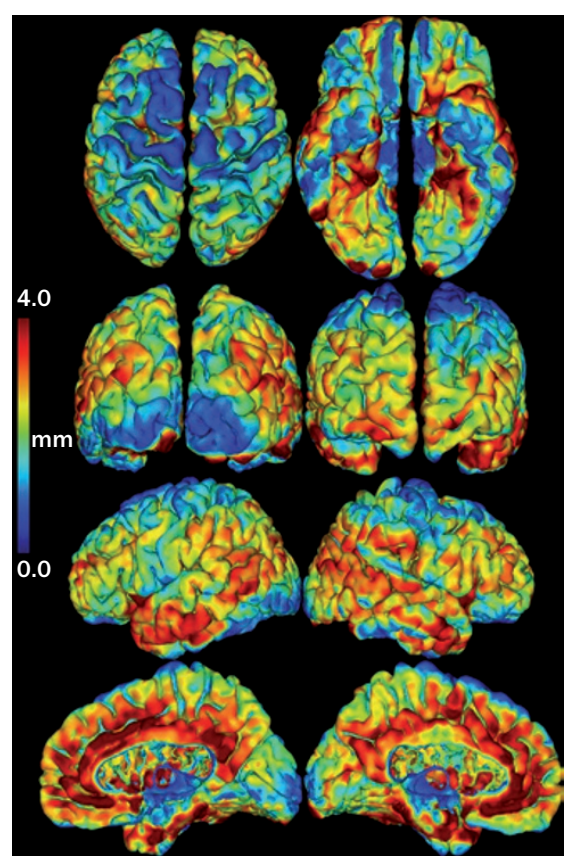

Cortical thickness distribution in a patient with chronic pancreatitis. Courtesy of J. B. Frøkjær. 